\title{
Thermodynamic Analysis of a Cascade Heat Pump Incorporated in High-Temperature Heating System
}

\author{
Nedžad Rudonja* - Milan Gojak - Ivan Zlatanović - Ružica Todorović \\ University of Belgrade, Faculty of Mechanical Engineering, Serbia
}

In this article is presented thermodynamic analysis of a cascade heat pump system designed for using in high-temperature heating systems. The own thermodynamic model was built by using properties of working fluids from the CoolProp base. The cascade heat pump was designed to use ambient air as heat source with temperature $t_{a m b}=-20{ }^{\circ} \mathrm{C}$ and for heating water in the high-temperature heating system up to $70{ }^{\circ} \mathrm{C}$. The projected heating capacity of the cascade heat pump was $100 \mathrm{~kW}$. The coefficient of performance (COP) of the cascade heat pump system due to use of different working mediums combinations in cycles of the cascade heat pump was investigated. For the best combination of working fluids (mediums) sub-cooling, super-heating, pressure loss in compressor's suction line, as well as exergy efficiency of the heat pump were analysed as a function of the mean temperature of the cascade heat exchanger.

Keywords: cascade heat pumps, working mediums, coefficient of performance, exergy efficiency

\section{Highlights}

- The influence of different combinations of working mediums on thermodynamic characteristics of the cascade heat pump was studied.

- The influence of the mean temperature of the cascade heat exchanger on COP was analysed and obtained thermodynamically the optimum value of the mean temperature.

- The impact of sub-cooling, super-heating, pressure loss in compressor's suction line and isentropic efficiency of compressor on COP were analysed.

- The exergy efficiency of the heat pump was calculated for the best combination of working mediums and obtained its dependence on the mean temperature of the cascade heat exchanger.

\section{INTRODUCTION}

Energy consumption permanently increases and consequently makes higher impact on environmental pollution and global warming [1]. Final energy consumption by end-users in residential sector was $25.4 \%$ in overall final energy consumption in Europe in 2015 [2]. By improving energy efficiency of systems and using renewable energy resources, such as biomass, geothermal energy, energy obtained from solar thermal and photovoltaic systems, both energy consumption and environmental pollution can be greatly reduced. Heat pumps as energy efficient systems are widely used because of their closely neutral impact on environmental and global warming [3].

The selection of heat pump type depends on heat source, temperature of both heat source and heat sink, as well as the temperature range in witch heat pump operates [4]. Serbia has huge potential for utilization of hydro-geothermal water as heat source [5], but utilization of those heat sources depends on location and for this reason in this article was analysed an air source heat pump for utilization in high-temperature heating systems. Although water and ground as heat sources are thermodynamically more favourable, those types of sources require high investment and exploitation costs. On the other hand, air as heat source is less thermodynamically unfavourable, but it is easy to use and available at all locations.

Traditional heat pumps are suitable for lowtemperature heating systems such as underfloor heating, low-temperature radiators or fan convection heaters [6]. When temperature range is too high, for instance, in high-temperature heating system, cascade heat pumps can be used as an economically acceptable solution [7] and better solution than single or two-stage heat pump [8] and [9]. Comparing to the single-stage heat pump, the compressors of a cascade heat pump has a smaller compression ratio and consequently heat pump achieves better performances.

In last decades, many researchers investigated various working mediums of heat pumps in order to improve their performance and consequently to maximize the coefficient of performance. In the article [10] was analysed several working mediums combinations in a cascade heat pump and thermodynamically the best case was with R600 in low-temperature (LT) circuit and R245fa in hightemperature (HT) circuit of the cascade heat pump (R600/R245fa). Ma et al. [11] analysed a high temperature cascade heat pump with R245fa (LT) 
and BY-3 (HT) as working mediums. They built the numerical model of the heat pump and validated model by own experimental results. $\mathrm{Xu}$ et al. [12] experimentally analysed a high temperature cascade heat pump for implementation in cold regions. The temperature of supply hot water was between $55^{\circ} \mathrm{C}$ and $75^{\circ} \mathrm{C}$, while ambient temperature was $-21^{\circ} \mathrm{C}$. As working medium in LT circuit was selected R404A, while in HT circuit it was R134a. The greatest COP, with water supply temperature near $55^{\circ} \mathrm{C}$, was 2.48 . Song et al. [13] and [14] studied the characteristics of the $\mathrm{CO}_{2}$ and R134a cycles in both combined and cascade system. The temperature of supply water was between $55^{\circ} \mathrm{C}$ and $75^{\circ} \mathrm{C}$, while ambient temperature was varied from $-20{ }^{\circ} \mathrm{C}$ up to $0{ }^{\circ} \mathrm{C}$. Bhattacharyya et al. [15] optimized the cascade system for refrigeration as well as for heating using combination R744/R290 as working mediums. In the article [16] a mathematical model was built to enable the prediction of optimal value of the mean temperature of cascade heat exchanger for combination R134a/R410A of a cascade heat pump. In the article [17] analysed the influence of the mean temperature of the cascade heat exchanger on performance of an air source cascade heat pump system. The mean temperature has been proven as a key factor in affecting the operating performance for heat pump system.

In literature so far there are few articles for cascade air source heat pump that operates at low temperature and provides high-temperature heating [11]. On the other hand, in this research was investigated the effect of different working mediums combinations on the heat pump performances. Four combinations of working mediums were analysed with aim to obtain the combination that gives the highest values of COP for given temperature range. Temperature range was selected in order to cover possibility of heating buildings with high temperature heating systems by using outdoor air as heat source. The COP was calculated for each combinations of working mediums by changing the mean temperature of the cascade heat exchanger. The condensing temperature in HT circuit was $75{ }^{\circ} \mathrm{C}$, while evaporating temperature in LT circuit was $-25^{\circ} \mathrm{C}$. The heating capacity of the cascade heat pump was $100 \mathrm{~kW}$. Furthermore, for the best combination of working mediums subcooling, super-heating, pressure loss in compressor's suction line, as well as exergy efficiency of the heat pump were analysed as a function of the mean temperature of the cascade heat exchanger. Obtained results are important for designers and engineers who design air source cascade heat pumps in given temperature range, because it was suggested the best combination of working mediums that gives the highest thermal performance of an air source cascade heat pump. Furthermore, scientists will have better insight of influence analyzed working phenomena on thermodynamic performances of cascade heat pump systems.

\section{THERMODYNAMIC MODEL}

A schematic diagram of an air source cascade heat pump system is presented in Fig. 1.

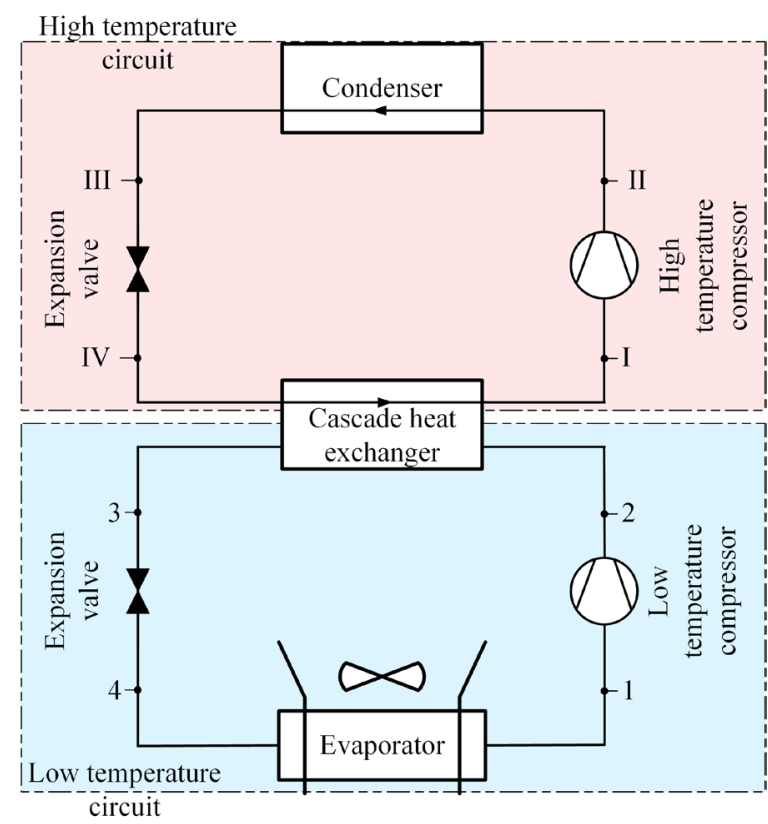

Fig. 1. An air source cascade heat pump

The heat pump consists of a low-temperature and a high-temperature circuits that are connected by a cascade heat exchanger. In this article all states of working mediums in low-temperature circuit are marked by arabic and in high-temperature circuit by roman numbers. Moreover, indexes of evaporating and condensing temperatures in LT circuit $\left(T_{e}, T_{c}\right)$ were lower letters and upper letters for HT circuit $\left(T_{E}\right.$, $\left.T_{C}\right)$.

Corresponding $T-S$ diagram of cycles of the cascade heat pump is shown in Fig. 2.

To simplify analysis in the article following assumptions were used:

- Heat transfer processes in all heat exchangers were isobaric;

- Heat transfer between surroundings and pipeworks was neglected;

- Pressure losses in all pipeworks were neglected; 


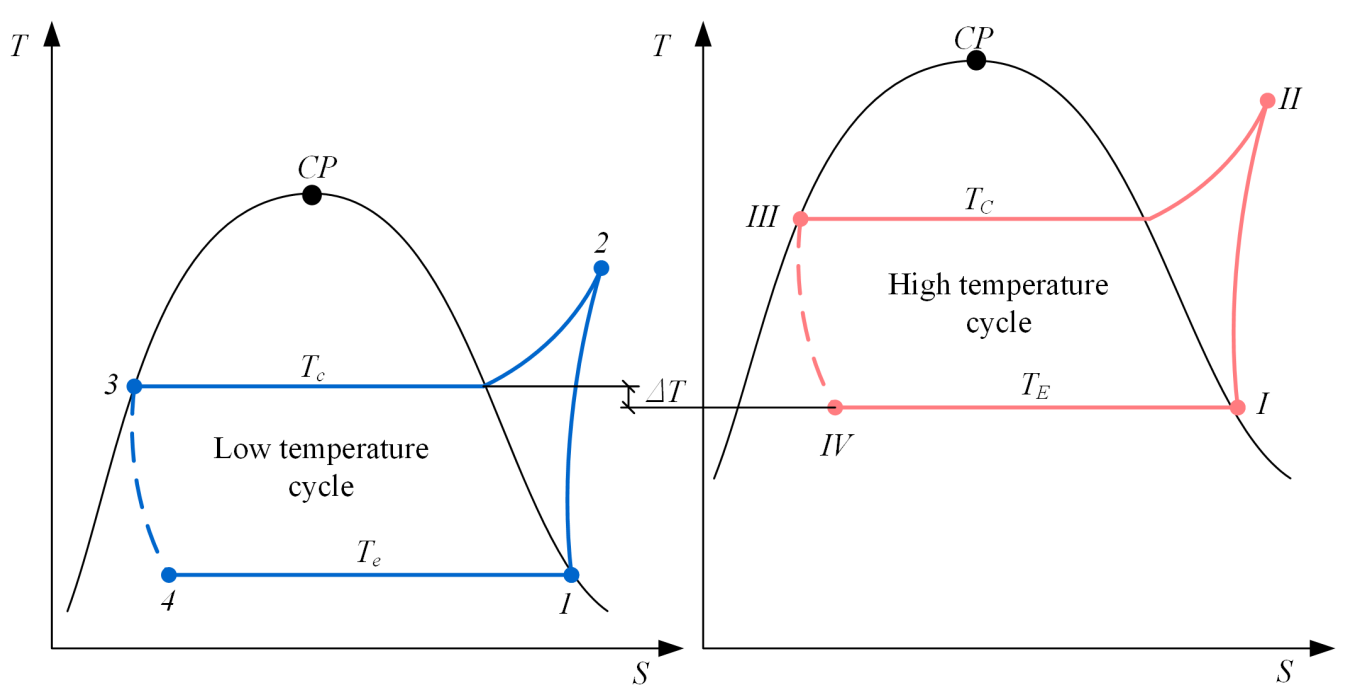

Fig. 2. T-S diagram of process of the cascade heat pump

- The difference between condensing temperature in LT and evaporating temperature in HT circuit was set at $\Delta T=7 \mathrm{~K}$;

- Evaporating temperature in LT circuit was $T_{e}=248 \mathrm{~K}$ (for $5 \mathrm{~K}$ lower than the ambient air temperature);

- Condensing temperature in HT circuit was $T_{C}=348 \mathrm{~K}$;

- Heating power of heat pump was $\dot{Q}_{\text {out }}=100 \mathrm{~kW}$.

The mean temperature of the cascade heat exchanger was defined as:

$$
T_{m}=\frac{T_{E}+T_{c}}{2}
$$

where $T_{E}$ and $T_{c}$ are evaporating temperature in HT and condensing temperature in LT circuit, respectively.

For given value of the mean temperature of the cascade heat exchanger, evaporating temperature in HT circuit as well as condensing temperature in LT circuit were calculated as:

$$
\begin{aligned}
& T_{E}=T_{m}-\frac{\Delta T}{2}, \\
& T_{c}=T_{m}+\frac{\Delta T}{2} .
\end{aligned}
$$

The mass flow rate in HT circuit was calculated as following:

$$
\dot{m}_{H T}=\frac{\dot{Q}_{o u t}}{h_{I I}-h_{I I I}},
$$

where $h_{I I}$ and $h_{I I I}$ are specific enthalpies of working medium in HT circuit at inlet and outlet of the condenser.
The power consumption of HT compressor is:

$$
P_{c, H T}=\dot{m}_{H T}\left(h_{I I}-h_{I}\right),
$$

where $h_{I}$ and $h_{I I}$ are specific enthalpies of working medium at inlet and outlet of HT compressor.

Based on introduced assumptions the heating capacity of LT circuit is equal to cooling capacity of HT circuit and follows:

$$
\dot{Q}_{\text {out }, L T}=\dot{Q}_{i n, H T}=\dot{Q}_{\text {out }}-P_{c, H T},
$$

where $\dot{Q}_{i n, H T}$ is cooling capacity of HT circuit.

The mass flow rate of working medium in LT circuit is:

$$
\dot{m}_{L T}=\frac{\dot{Q}_{o u t, L T}}{h_{2}-h_{3}},
$$

where $h_{2}$ and $h_{3}$ are specific enthalpies of working medium at inlet and outlet of LT condenser.

The power consumption of LT compressor is:

$$
P_{c, L T}=\dot{m}_{L T}\left(h_{2}-h_{1}\right),
$$

where $h_{1}$ and $h_{2}$ are specific enthalpies of working medium at inlet and outlet of LT compressor.

The COP of the cascade heat pump was calculated as:

$$
C O P_{H P}=\frac{\dot{Q}_{\text {out }}}{P_{c, L T}+P_{c, H T}} .
$$

Exergy efficiency (the second-law efficiency) is measure of perfection of a system in given working conditions. It can be defined as the ratio of the thermal efficiency of the actual process compared to the reversible process. For heat pumps, exergy efficiency 
can be defined as the ratio of the $C O P$ of actual heat pump cycle to $C O P$ of Carnot heat pump cycle, that operate between the same temperatures of heat source and heat sink [18]:

$$
\eta_{e x}=\frac{C O P_{H P}}{C O P_{\text {Carnot }}}
$$

The isentropic efficiency of a compressor is defined as the ratio of the work input to an isentropic process to the work input to the actual process, which takes place between the same inlet and exit pressures. The isentropic efficiency of both compressors was same and defined as:

$$
\eta_{c}=\frac{h_{\text {out }, i}-h_{\text {in }}}{h_{\text {out }}-h_{\text {in }}}
$$

where $h_{\text {out }, i}$ is specific enthalpy of the working medium at outlet of the compressor in the case of its isentropic compression.

\section{RESULTS AND DISCUSSION}

\subsection{Working Mediums Selection - Basic Thermodynamic cycle}

The first step of thermodynamic analysis, for given working conditions of the cascade heat pump, was conducted in order to obtain the best combination of working mediums in LT and HT circuits. Based on literature review, the next combinations of working mediums were used: R717 - R245fa, R245fa - R717, R134a - R600 and R1270 - R717. The results of conducted analyses are shown in Fig. 3. During simulations the mean temperature of the cascade heat exchanger was varied for all working mediums combinations and it was obtained that R245fa in LT and R717 in HT give the maximum value of COP. In this case the isentropic efficiency of compressors was $\eta_{c}=1.0$, the cycles were without sub-cooling, superheating and pressure loses at compressor's suction line.

Based on Fig. 3 it can be also concluded that the maximum value of $C O P$ for the combination $\mathrm{R} 245 \mathrm{fa} /$ R717 was obtained for the mean temperature of cascade $21{ }^{\circ} \mathrm{C}$ as well as that for different working mediums combinations there is different value of the mean temperature of the cascade heat exchanger which gives maximum value of $C O P$.

The next steps of thermodynamic analysis were conducted for the best working mediums combination R245fa/R717 and for thermodynamic cycles modified in accordance with the considered impacts.

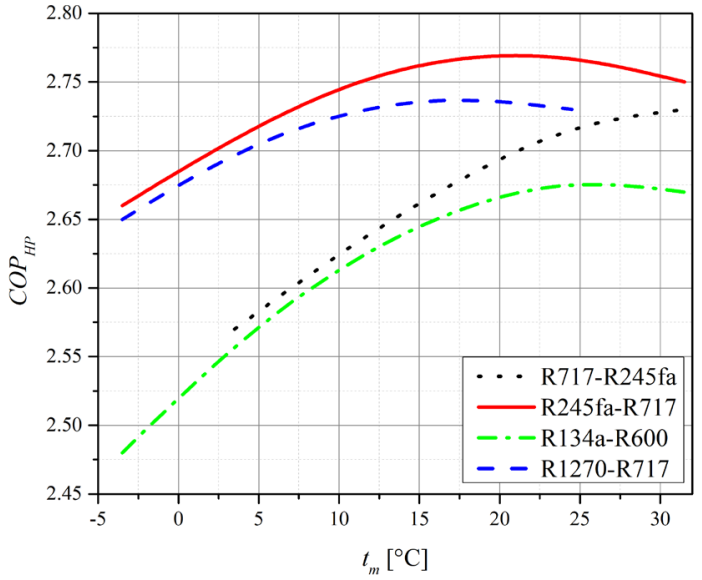

Fig. 3. COP dependence on working mediums combinations and the mean temperature of the cascade heat exchanger

\subsection{The Impact of Sub-cooling}

The influence of sub-cooling on COP is shown in Fig. 4. In this case the isentropic efficiency of the compressors was $\eta_{c}=1.0$, the cycles were without super-heating and pressure loses at compressor's suction line.

In this case the temperature at outlet of the condenser was calculated using temperature of condensing in LT, i.e. in HT circuit as follows:

$$
\begin{gathered}
T_{3}=T_{c}-\Delta T_{s c}, \\
T_{I I I}=T_{C}-\Delta T_{s c},
\end{gathered}
$$

where $\Delta T_{s c}$ is difference between corresponding the condensing temperature and the temperature at outlet of the condenser. In this case $\Delta T_{s c}$ was same for both LT and HT circuit.

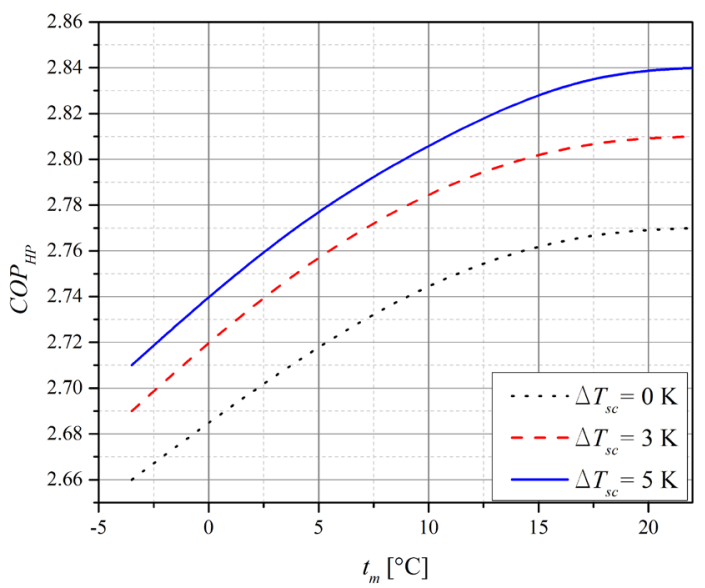

Fig. 4. COP dependence on the mean temperature of the cascade heat exchanger and the temperature of sub-cooling 
From Fig. 4 it can be concluded that sub-cooling working medium for $5 \mathrm{~K}$ gives the increase in $\mathrm{COP}$ value for approximately $2.5 \%$.

The Experimental section should provide details of the experimental set-up and the methods used to obtain the results. To make this section interesting, explain the choices you made in your experimental procedure. This section should provide sufficient detail for other scientists to be able to reproduce the experiments presented in this paper.

\subsection{The Impact of Super-heating}

The influence of super-heating of working medium at inlet of the compressor on $C O P$ is shown in Fig. 5. In this case the isentropic efficiency of the compressors was $\eta_{c}=1.0$, the cycles were without sub-cooling and pressure loses at compressor's suction line. In this case the temperature at inlet of the compressor was calculated using the temperature of evaporating in LT, i.e. in HT circuit as follows:

$$
\begin{aligned}
& T_{1}=T_{e}+\Delta T_{s h}, \\
& T_{I}=T_{E}+\Delta T_{s h},
\end{aligned}
$$

where $\Delta T_{s h}$ was same for both LT and HT circuit.

From Fig. 5 it can be concluded that super-heating has small influence on $C O P$, but it is still important from the point of view of compressor protection.

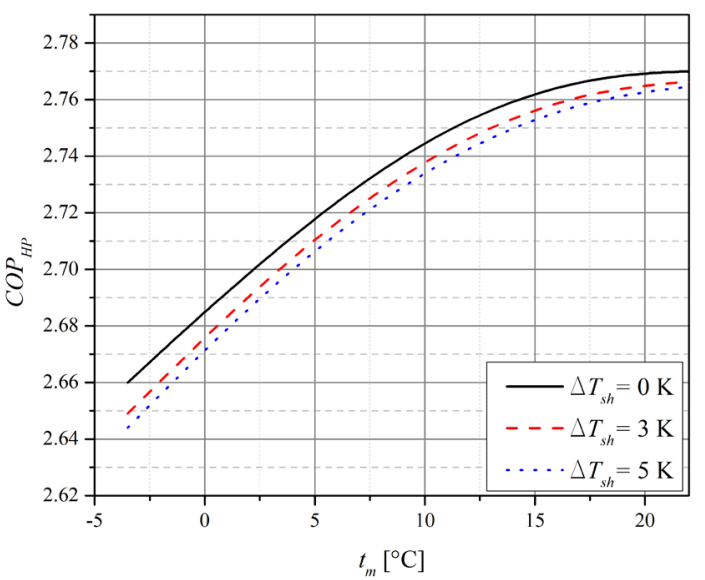

Fig. 5. COP dependence on the mean temperature of the cascade heat exchanger and the temperature of super-heating

\subsection{The Impact of Pressure Looses at Compressor's Suction line}

The influence of pressure loses at suction line of the compressor on $C O P$ for selected working mediums is shown in Fig. 6.

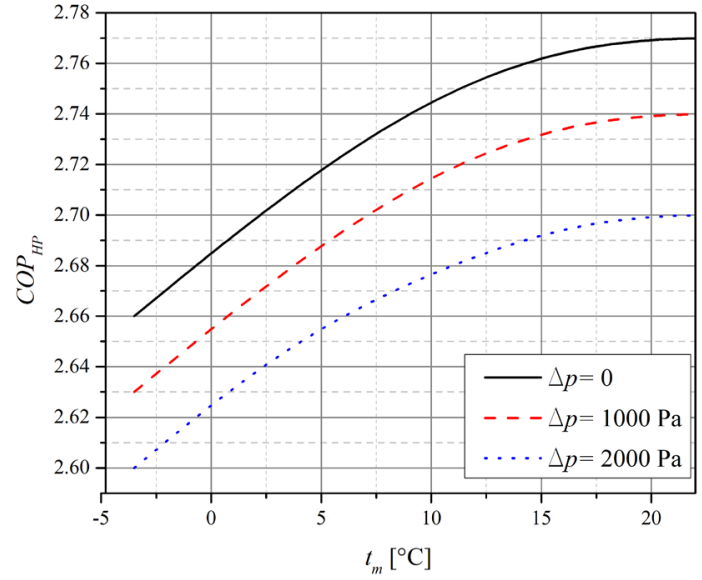

Fig. 6. COP dependence on the mean temperature of the cascade heat exchanger and the loss of pressure at the compressor suction line

In this case the isentropic efficiency of the compressors was was $\eta_{c}=1.0$, the cycles were without sub-cooling and super-heating. The pressure of working mediums at inlet of the compressors were calculated as following:

$$
\begin{aligned}
& p_{1}=p_{e}-\Delta p, \\
& p_{I}=p_{E}-\Delta p,
\end{aligned}
$$

where $p_{e}$ and $p_{E}$ are functions of corresponding evaporating temperatures, i.e. $p_{e}=f\left(T_{e}\right)$ and $p_{E}=f\left(T_{E}\right)$. It can be concluded that pressure drop of $2000 \mathrm{~Pa}$ at the suction line produces the decrease in COP by approximately $2.5 \%$.

\subsection{The Impact of Isentropic Efficiency of the Compressors}

The influence of isentropic efficiency of the compressors on COP depending on the mean temperature of the cascade heat exchanger is shown in Fig. 7.

In this case the cycles were without sub-cooling, super-heating and pressure loses at compressor's suction line. As expected, it was shown that isentropic efficiency of the compressor $\left(\eta_{c}\right)$ has considerable impact on $C O P$ and that dependence is a linear function Fig. 8, where maximum value of $C O P$ was obtained for optimum value of the mean temperature of the cascade heat exchanger $\left(21^{\circ} \mathrm{C}\right)$.

\subsection{Exergy Efficiency}

The maximum value of the exergy efficiency of the cascade heat pump for selected working mediums was calculated according Eq. (10) and obtained values 


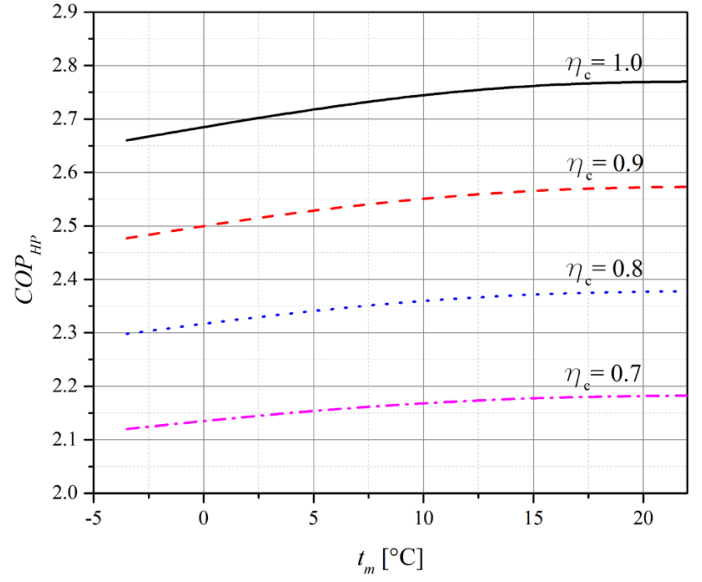

Fig. 7. COP dependence on the mean temperature of the cascade heat exchanger and the isentropic efficiency of the compressors

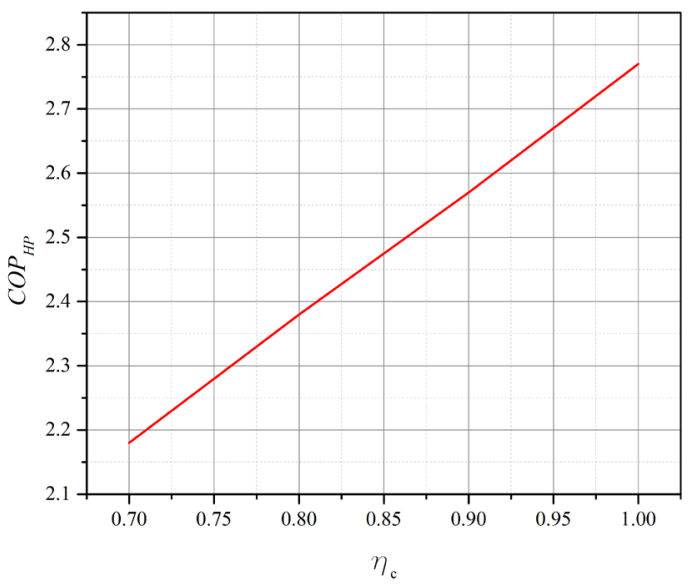

Fig. 8. The maximum COP value depending on the isentropic efficiency of the compressors

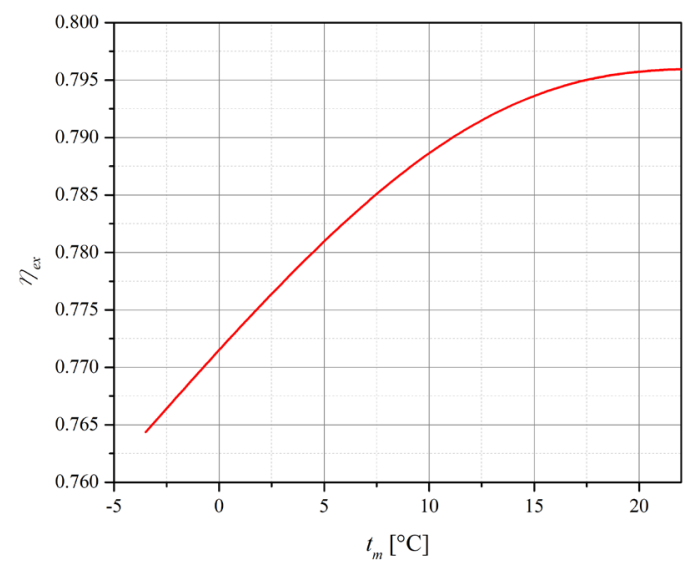

Fig. 9. The exergy efficiency of the cascade heat pump depending on the mean temperature of the cascade heat exchanger

are presented in Fig. 9. In this case the isentropic efficiency of the compressors was $\eta_{c}=1.0$, the cycles were without sub-cooling, super-heating and pressure loses at compressor's suction line.

For analysed the cascade heat pump, for given assumptions, the maximum value of the exergy efficiency was about 0.796 which obtained for optimum value of the mean temperature of the cascade heat exchanger $\left(21^{\circ} \mathrm{C}\right)$. The character of dependence is same as the dependence of $C O P$.

\section{CONCLUSIONS}

A cascade heat pump system can be used as heat source of high temperature heating systems. The combination of working mediums is very important from point of view energy efficiency of heat pump system. For given working conditions the best combination of working mediums was R245fa in lowtemperature circuit and R717 in high-temperature heat pump circuit. The maximum value of the $C O P$ was 2.77 and it can be concluded that use of cascade heat pumps in high-temperature heating systems is justified. For each combination of working mediums there is the optimal value of the mean temperature of the cascade heat exchanger that gives maximum value of COP. The pressure loses at compressor's suction line by $2000 \mathrm{~Pa}$ contributes to decreasing in $C O P$ for closely $2.5 \%$. Sub-cooling working mediums at outlet of the condenser for $5 \mathrm{~K}$ gives the increase of $C O P$ value approximately by $2.5 \%$. Super-heating of working mediums at the compressor inlet is important for compressor protection but has a small and negative impact on $C O P$ value. Decreasing of the isentropic efficiency of the compressors significantly decreases $C O P$ value as well as linearly impacts on it.

\section{ACKNOWLEDGEMENTS}

The research was conducted as a part of the agreement on realization and financing of scientific research work in 2020 between the Ministry of Education, Science and Technological Development of the Republic of Serbia and the Faculty of Mechanical Engineering in Belgrade - contract number: 451-03-68 / 2020-14 / 200105.

\section{REFERENCES}

[1] Bilgen, S., (2014). Structure and environmental impact of global energy consumption. Renewable and Sustainable Energy Reviews, vol.38, p. 890-902, D0l:10.1016/j. rser.2014.07.004.

[2] European Commision (2015). Energy, transport and environment - statistics on three closely related domains, from: 
https://ec.europa.eu/eurostat/statistics-explained/index. php?title=File:Final_energy_consumption,_EU-28,_2015_ (\%25_of_total,_based_on_tonnes_of_oil_equivalent).png, accessed on 2020-06-01.

[3] Hammond, G.P., Norman, J.B. (2014). Heat recovery opportunities in UK industry, Applied Energy, vol. 116, p. 387397, D0I:10.1016/j.apenergy.2013.11.008.

[4] Boahen, S., Anka, S.K., Lee, K.H., Choi, J.M. (2021). Performance analysis of cascade multi-functional heat pump in summer season, Renewable Energy, vol. 163, p. 10011011, D0I:10.1016/j.renene.2020.09.036.

[5] Martinović, M., Andrjević, S., Saljnikov, A., Komatina, M., Rudonja, N., Stevanović, Z. (2008). Hydrogeothermal resources \& heat pumps - district heating alternative of Serbia. KGH Conference Proceedings, p. 314-320.

[6] Ivanovski, I., Goričanec, D., Salamunić, J., Žagar, T. (2018). The comparison between two high-temperature heat-pumps for the production of sanitary water. Strojniški vestnik - Journal of Mechanical Engineering, vol. 64, no. 7-8, p. 437-442, DOI:10.5545/sv-jme.2017.5082.

[7] Goričanec, D. Rudonja, N., Komatina, M., Andrejević, S., Krope, J., Zlatanović, I. (2008). Cascade type geothermal heat pump - economic analysis and environmental impact, $\mathrm{KGH}$ Conference Proceedings, p. 140-146.

[8] Bertsch, S.S., Groll, E.A. (2008). Two-stage air-source heat pump for residential heating and cooling applications in northern U.S. climates. International Journal of Refrigeration, vol. 31, no. 7, p. 1282-1292, D0l:10.1016/j. jjrefrig.2008.01.006.

[9] Jung, H.W., Kang, H., Yoon, W.J., Kim, Y. (2013). Performance comparison between a single-stage and a cascade multifunctional heat pump for both air heating and hot water supply. International Journal of Refrigeration, vol. 36, no. 5, p. 1431-1441, D0I:10.1016/j.jijrefrig.2013.03.003.

[10] Uusitalo, A., Turunen-Saaresti, T., Honkatukia, J., Tiainen, J., Jaatinen-Värri, A. (2020). Numerical analysis of working fluids for large scale centrifugal compressor driven cascade heat pumps upgrading waste heat. Applied Energy, vol. 269, art. ID 115056, DOI:10.1016/j.apenergy.2020.115056.
[11] Ma, X., Zhang, Y., Fang, L., Yu, X., Li, X, Sheng, Y., Zhang, Y. (2008). Performance analysis of a cascade high temperature heat pump using R245fa and BY-3 as working fluid. Applied Thermal Engineering, vol. 140, p. 466-475, D0I:10.1016/j. applthermaleng.2018.05.052.

[12] Xu, L., Li, E., Xu, Y., Mao, N., Shen, X., Wang, X. (2020). An experimental energy performance investigation and economic analysis on a cascade heat pump for high-temperature water in cold region. Renewable Energy, vol. 152, p. 674-683, DOI:10.1016/j.renene.2020.01.104.

[13] Song, Y., Li, D., Cao, F., Wang, X. (2017). Theoretical investigation on the combined and cascade C02/R134a heat pump systems for space heating. Applied Thermal Engineering, vol. 124, p. 1457-1470, D0l:10.1016/j. applthermaleng.2017.06.014.

[14] Song, Y., Li, D., Yang, D., Jin, L., Cao, F., Wang, X. (2017). Performance comparison between the combined R134a/CO2 heat pump and cascade R134a/CO2 heat pump for space heating. International Journal of Refrigeration, vol. 74, p. 592605, D0I:10.1016/j.ijrefrig.2016.12.001.

[15] Bhattacharyya, S., Garai, A., Sarkar, J. (2009). Thermodynamic analysis and optimization of a novel N20-CO2 cascade system for refrigeration and heating. International Journal of Refrigeration, vol. 32, no. 5, p. 1077-1084, D0l:10.1016/j. ijrefrig.2008.09.008.

[16] Park, H., Kim, D.H., Kim, M.S. (2013). Thermodynamic analysis of optimal intermediate temperatures in R134aR410A cascade refrigeration systems and its experimental verification. Applied Thermal Engineering, vol. 54, no. 1, p. 319-327, D0l:10.1016/j.applthermaleng.2013.01.005.

[17] Wang, W., Zhou, Q., Tian, G., Hu, B., Li, Y., Cao, (2020). F. The intermediate temperature optimization for cascade refrigeration system and air source heat pump via extreme seeking control. International Journal of Refrigeration, vol. 117, p. 150-162, D0I:10.1016/j.jirefrig.2020.05.007.

[18] Balmer, R.T. (2011). Modern Engineering Thermodynamics, Elsevier, Amsterdam, DOl:10.1016/B978-0-12-3749963.00028-2. 